\title{
A search for dormant binaries with degenerate components in $\omega$ Centauri and NGC 6397
}

\author{
M. Rozyczka ${ }^{1}$, J. Kaluzny ${ }^{1}$, P. Pietrukowicz ${ }^{1,2}$, W. Pych $^{1}$, M. Catelan ${ }^{2}$, C. Contreras ${ }^{2}$, and I. B. Thompson ${ }^{3}$ \\ ${ }^{1}$ Nicolaus Copernicus Astronomical Center, ul. Bartycka 18, 00-716 Warszawa, Poland \\ e-mail: mnr@camk.edu.pl \\ 2 Pontificia Universidad Católica de Chile, Departamento de Astronomía y Astrofísica, Av. Vicuña MacKenna 4860, $782-0436$ Macul, \\ Santiago, Chile \\ 3 Carnegie Institution of Washington, 813 Santa Barbara Street, Pasadena, CA 91101, USA
}

Received 29 June 2010 / Accepted 13 September 2010

\begin{abstract}
Aims. We report on the first spectroscopic search for quiescent degenerate binaries in globular clusters.

Methods. Our survey is based on a sample of short-period optical variables that are likely optical counterparts of the quiescent X-ray sources in $\omega$ Centauri (NGC 5139) and NGC 6397.

Results. The studied candidates have nearly sinusoidal light curves with amplitudes of $0.05-0.12$ mag in $V$ ( 0.35 mag in one case) and periods of $0.1-1.3$ days. This type of variability, most probably from the ellipsoidal effect, has been observed in X-ray novae when they settled into quiescence after an outburst.

Conclusions. We find that two of the surveyed systems harbor dim components with masses in excess of $1 M_{\odot}$, making them attractive targets for future investigations. We also suggest that there are two subpopulations of blue stragglers in $\omega$ Centauri, differing in either mass-transfer history or helium content, or even in both at once.
\end{abstract}

Key words. stars: general - binaries: close - blue stragglers - subdwarfs - globular clusters: individual: NGC 5139 ( $\omega$ Centauri) globular clusters: individual: NGC 6397

\section{Introduction}

Since the early surveys with the Uhuru and OSO-7 satellites, it has been known that X-ray sources occur about a thousand times more frequently in globular clusters (GCs) than in the rest of the Galaxy (e.g., Katz 1975; Clark 1975). Over fifteen hundred of them have been detected to date in these stellar systems by recent missions such as Chandra and XMM-Newton (Pooley 2010, and references therein). In addition, over a hundred millisecond pulsars - objects that have evolved in low-mass X-ray binaries - are known to reside in GCs (Lynch et al. 2010). Most of the X-ray sources are binaries with degenerate components (neutron stars or white dwarfs), henceforth referred to as "degenerate binaries". Remarkably, no stellar-mass black holes (BH) are known in Galactic GCs, despite theoretical predictions that they should exist (e.g., Devecchi et al. 2007). The presence of intermediatemass black holes (IMBHs) $\left(100 M_{\odot}<M<10^{3-4} M_{\odot}\right)$ in GCs has also not been proven yet (e.g., Maccarone \& Servillat 2008; van der Marel \& Anderson 2010).

While the origin of IMBHs is unclear, stellar-mass black holes should form along with neutron stars via supernova explosions during the early evolution of a cluster. The very presence of such objects would provide interesting information concerning the effectiveness of dynamical interactions leading to the ejection of $\mathrm{BHs}$ into the intracluster medium, which according to the predictions of current approximate models should be very high (e.g., Downing et al. 2010). Their absence would confirm those predictions. Alternatively, it could mean that massive lowmetallicity stars cannot produce BHs, which would have important implications for the origin of early BHs that are believed to be seeds for the first galaxies in the Universe. In either case, searching for black holes in GCs is certainly a worthwhile task.

It is well known that field X-ray novae (binaries most probably hosting stellar-mass black holes) spend most of their time in quiescence, showing only ellipsoidal variability in the visible domain (Remillard \& McClintock 2006). Since GCs harbor a multitude of degenerate binaries, they must also contain many systems that presently accrete at a very low rate or do not accrete at all, thus being very weak or undetectable in the X-rays. Based on X-ray luminosity $\left(L_{\mathrm{X}}\right)$ and hardness ratio, Grindlay (2006) splits the population of weak X-ray sources in GCs into four major classes. The first three, arranged according to $L_{X}$ increasing from $10^{29}$ (the present sensitivity limit for the nearest GCs) to $10^{32} \mathrm{erg} \mathrm{s}^{-1}$, are i) active binaries (binary mainsequence stars in which the X-ray emission originates from chromospheric activity, e.g. BY Dra type systems); ii) cataclysmic variables (in which a white dwarf accretes from a low-mass main-sequence companion); iii) quiescent low-mass X-ray binaries (in which a neutron star intermittently accretes from a main-sequence or evolved companion), hereafter referred to as qLMXBs. The fourth class is composed of millisecond pulsars, whose luminosity strongly depends on the predominant mechanism generating X-ray quanta (residual accretion, collision of the pulsar wind with the ambient medium or with matter lost by the companion, thermal surface emission).

The study of weak X-ray sources is hampered by how, even in the nearest GCs they are detected down to the present sensitivity limit and often remain unclassified, as they are too dim to allow for an estimate of the hardness ratio. Most of them are likely active binaries or cataclysmic variables, but some may be 
quiescent systems with neutron stars or even black holes. Optical counterparts of these systems are often weak and hard to identify in the crowded GC environment (e.g., Verbunt et al. 2008). So far, the X-ray data have allowed identifying a few candidate qLMXBs, but their nature has not been confirmed by optical spectroscopy (Guillot et al. 2009).

We decided to follow an entirely different approach. Instead of looking for optical counterparts of X-ray sources, we selected a sample of candidate systems for degenerate binaries from short-period, low-amplitude optical variables cataloged in existing surveys and measured their radial velocities. Our targets have nearly sinusoidal light curves with $V$-band amplitudes smaller than $0.35 \mathrm{mag}$ and periods shorter than $\sim 1.3$ days. This type of variability is common in all classes of close degenerate binaries; in particular, it is observed in optical counterparts of several Galactic X-ray novae that almost certainly harbor BHs (Remillard \& McClintock 2006). The optical modulation is induced mostly by the ellipsoidal effect from the nondegenerate component, with an amplitude depending mainly on the Roche lobe filling factor and the inclination of the orbit. Close degenerate binaries can be unambiguously identified as single-line systems with high orbital velocities $\left(K>150 \mathrm{~km} \mathrm{~s}^{-1}\right)$. Among them, those harboring a black hole are distinguished by a mass function $f_{m}=(m \sin i)^{3} /\left(m_{\text {bin }}\right)^{2}>2 M_{\odot}$, where $m_{\text {bin }}$ and $m$ stand for the total mass of the binary and the mass of one of its components (Remillard \& McClintock 2006).

Our sample and observational data are introduced in Sect. 2. A detailed analysis of the data is reported in Sect. 3, and the results are discussed in Sect. 4.

\section{Observations and data reduction}

Optical counterparts of active X-ray sources in GCs have been found essentially everywhere in color-magnitude diagrams: to the left of the main sequence, on the main sequence, to the right of it, on subgiant, giant, and horizontal branches, and also above the main-sequence turnoff in the regions occupied by blue stragglers and EHB stars (e.g. Heinke et al. 2005; Servillat et al. 2008). Thus, while preparing this study we felt it justified to pick photometrically suitable targets without paying attention to their location with respect to the main sequence. Since largeamplitude variables are more likely to be ordinary contact binaries, we avoided objects with full $V$-band amplitudes in excess of $\sim 0.35 \mathrm{mag}$. Suspected pulsating variables were excluded and we took care to exclude systems with appreciable X-ray emission. Where it was possible, the membership status of the target was established based on the data of Bellini et al. (2009) for $\omega$ Cen, and Anderson (priv. comm.) and Strickler et al. (2009) for NGC 6397. In the remaining cases membership was decided a posteriori based on the systemic velocity with respect to the cluster.

Alltogether, we selected seven objects in $\omega$ Cen and four in NGC 6397. In color-magnitude diagrams two of them are located to the left of the main sequence, seven above the mainsequence turnoff, and two at the turnoff itself (see Figs. 1 and 2). A summary of their basic data is given in Table 1, where the labels are the same as those introduced by Kaluzny et al. (2004) for $\omega$ Cen and Kaluzny et al. (2006) for NGC 6397.

Our paper is based on photometric measurements of Kaluzny et al. (2004, 2006), supplemented by those of Weldrake et al. (2007) and by our unpublished data for NGC 6397 obtained on the du Pont telescope at Las Campanas Observatory, Chile. A total of $771 \mathrm{~V}$-band frames was acquired between 2009 June 20

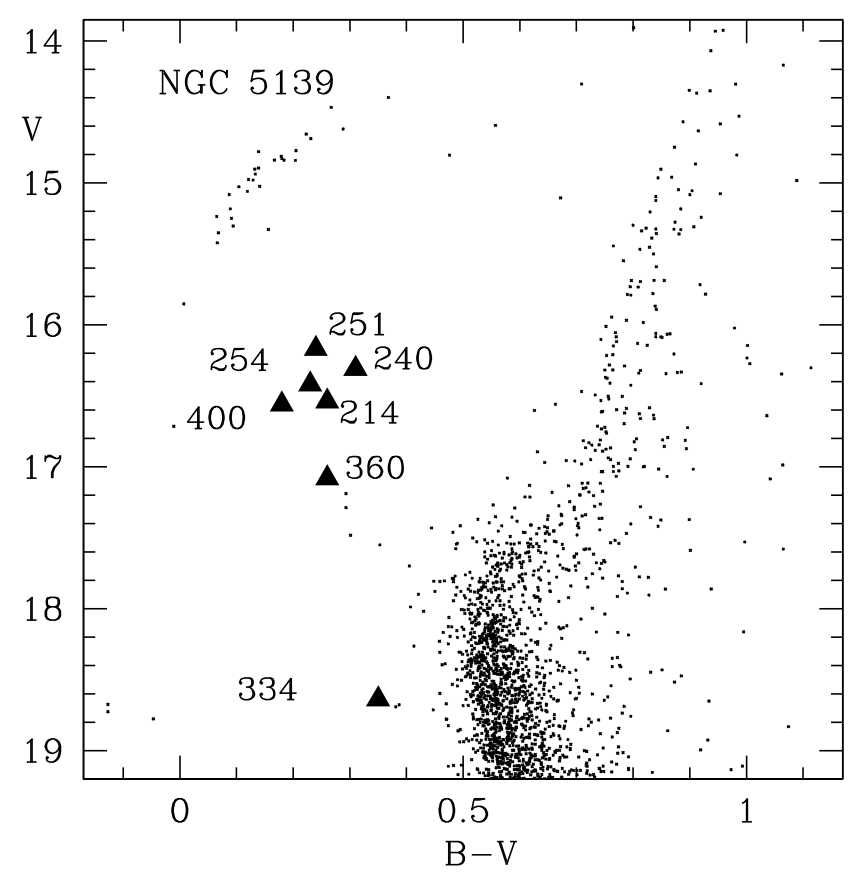

Fig. 1. Color-magnitude diagram of $\omega$ Cen based on CASE data (Kaluzny et al. 2004) transformed to the BV system with the help of standards listed by Stetson (2000). Marked are the locations of the investigated systems.

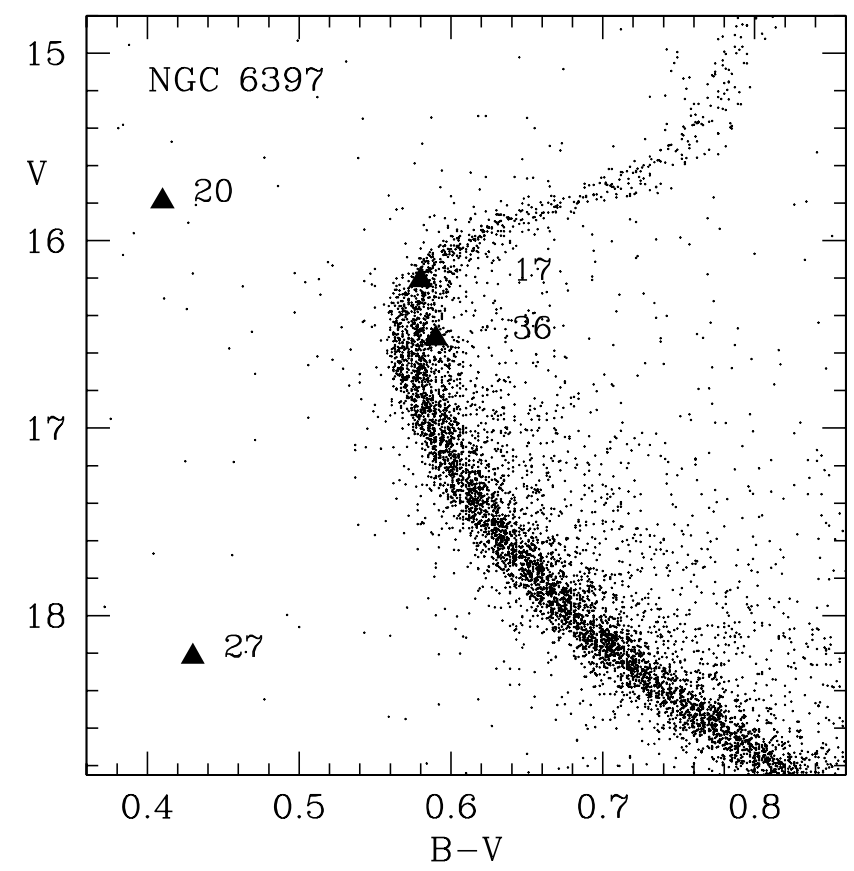

Fig. 2. Color-magnitude diagram of NGC 6397 (after Kaluzny et al. 2006) with marked positions of the investigated systems.

and June 30, and reduced in the same way as in Kaluzny et al. (2006). The newer data demonstrated that the observed light variations were coherent, although in some cases the light curve was changing from symmetric to asymmetric or vice versa.

Spectroscopic data were collected during the nights 20/21 and 21/22 of May 2009 with the MagE (Magellan Echellette) spectrograph attached to the 6.5-m Magellan-Clay telescope at 
Table 1. Basic information on the target objects.

\begin{tabular}{lcccccccc}
\hline \hline Cluster & Star & $\begin{array}{c}\alpha_{2000} \\
{[\mathrm{~h}: \mathrm{m}: \mathrm{s}]}\end{array}$ & $\begin{array}{c}\delta_{2000} \\
{\left[:^{\circ}::^{\prime \prime}\right]}\end{array}$ & $p_{\mathrm{m}}{ }^{*}$ & $\begin{array}{c}L_{\mathrm{X}}{ }^{* *} \\
{\left[10^{30} \mathrm{erg} \mathrm{s}^{-1}\right]}\end{array}$ & $\begin{array}{c}V \\
{[\mathrm{mag}]}\end{array}$ & $\begin{array}{c}B-V \\
{[\mathrm{mag}]}\end{array}$ & Ref. $^{\dagger}$ \\
\hline$\omega$ Cen & V214 & $13: 27: 21.82$ & $-47: 37: 19.0$ & 97 & $<13$ & 16.55 & 0.25 & 1,2 \\
$\omega$ Cen & V240 & $13: 27: 28.68$ & $-47: 26: 19.5$ & 99 & $<1.2$ & 16.31 & 0.30 & 1,2 \\
$\omega$ Cen & V251 & $13: 27: 28.02$ & $-47: 26: 43.7$ & 97 & $<1.2$ & 16.17 & 0.25 & 1 \\
$\omega$ Cen & V254 & $13: 27: 28.61$ & $-47: 27: 39.0$ & 99 & $<1.2$ & 16.41 & 0.25 & 1,2 \\
$\omega$ Cen & NV334 & $13: 27: 23.39$ & $-47: 29: 11.9$ & 88 & $<1.2$ & 18.63 & 0.35 & 1 \\
$\omega$ Cen & NV360 & $13: 26: 02.06$ & $-47: 32: 23.6$ & 100 & $<1.2$ & 17.09 & 0.25 & 1 \\
$\omega$ Cen & NV400 & $13: 26: 10.09$ & $-47: 31: 50.3$ & 99 & $<1.2$ & 16.56 & 0.20 & 1 \\
NGC 6397 & V17 & $17: 40: 43.78$ & $-53: 41: 16.2$ & 100 & 0.4 & 16.17 & 0.40 & 3,4 \\
NGC 6397 & V20 & $17: 40: 41.66$ & $-53: 40: 33.1$ & 100 & 1.6 & 15.75 & 0.40 & 3,4 \\
NGC 6397 & V27 & $17: 41: 13.80$ & $-53: 41: 14.1$ & - & - & 18.19 & 0.45 & 3,4 \\
NGC 6397 & V36 & $17: 40: 44.10$ & $-53: 42: 11.3$ & - & 0.4 & 16.48 & 0.60 & 3,4 \\
\hline
\end{tabular}

Notes. ${ }^{(*)}$ Membership probability: Bellini et al. (2009) for V214-NV400; Strickler et al. (2009) and Anderson (2010, priv. comm.) for V17-V20; no data available for V27 and V36. ${ }^{(*)}$ X-ray luminosity: Gendre et al. (2003) for V214; Haggard et al. (2004) for V240-NV400; Bogdanov et al. (2010) for V17-V20 and V36; no data available for V27. ${ }^{(\dagger)}$ Photometry: 1 - Kaluzny et al. (2004); 2 - Weldrake et al. (2007); 3 - Kaluzny et al. (2006); 4 - our unpublished data collected in 2009.

Las Campanas Observatory. The seeing varied between 0.'5 and $0 . ' 8$ on the first night, and betweeen 0.4 and 0.9 on the second one. A 0. '85 slit was used, providing a resolution $R=4820$.

During the observations, pairs of scientific spectra taken for the same target were separated by an exposure of a thoriumargon hollow-cathode lamp. The exposure times per spectrum ranged from $120 \mathrm{~s}$ to $1080 \mathrm{~s}$, depending on object brightness and observing conditions. After bias and flat-field correction, each pair of frames was combined into a single frame, allowing for the rejection of cosmic ray hits. The observations were reduced with the IRAF ${ }^{1}$ ECHELLE package.

To determine the mass function of a given target it was sufficient to take just a few spectra at different phases, and fit a simple sinusoid to its velocity curve phased with the photometric ephemeris. Altogether, 96 spectra were obtained (from 5 to 10 per object). The useful range of the reduced spectra extended from $4050 \AA$ to $6800 \AA$, where the spectra had $13<S / N<23$ (with a few cases of lower quality). Radial velocities were measured in that range with the help of IRAF routines FXCOR and XCSAO, using synthetic templates from the library compiled by Munari et al. (2005). The results were verified by applying the broadening function formalism described by Rucinski (2002). In most cases the differences between various measurements were smaller than $\Delta v=10 \mathrm{~km} \mathrm{~s}^{-1}$, which may be regarded as a fair estimate of the error.

\section{Analysis}

While our data are insufficient for a detailed analysis, an estimate of the basic parameters of the observed systems is possible. In all cases but one (V20 in NGC 6307) single-line spectra are observed, indicating that to a first approximation all the light we receive from the binary is produced by just one of the components. Based on this assumption, we may set some limits for acceptable configurations.

\subsection{Method}

Our observables are orbital period $P$, apparent $V$-band magnitude $V$, color index $B-V$, amplitude of the light curve $\Delta V_{\text {obs }}$, and

\footnotetext{
${ }^{1}$ IRAF is distributed by the National Optical Astronomy Observatories, which are operated by the AURA, Inc., under cooperative agreement with the NSF.
}

amplitude of the velocity curve $K_{\text {obs }}$. Since the distance moduli to $\omega$ Cen and NGC 6397 are known, the apparent $V$-magnitude of a system belonging to the cluster is equivalent to the observed bolometric magnitude of its visible component $M_{\text {bol }}^{\mathrm{o}}$ ("observed" here means "derived from observations"; hence index "o"). We adopt $(m-M)_{0}=13.75 \mathrm{mag}$ and $E(B-V)=0.115 \mathrm{mag}$ for $\omega$ Cen (Villanova et al. 2007, and references therein), along with $(m-M)_{0}=12.03 \mathrm{mag}$ and $E(B-V)=0.183 \mathrm{mag}$ for NGC 6397 (Richer et al. 2008, and references therein). Assuming $A_{V}=3.1 E(B-V)$, the corresponding values of $A_{V}$ are $0.36 \mathrm{mag}$ and $0.57 \mathrm{mag}$, yielding a distance modulus $(m-M)_{V}$ of $14.11 \mathrm{mag}$ for $\omega$ Cen and $12.60 \mathrm{mag}$ for NGC 6397.

The effective temperature of the visible component $T$ is known from the relation between the color index $B-V$ and temperature derived for an appropriate chemical composition. We adopt $[\mathrm{Fe} / \mathrm{H}]=-1.8$ for $\omega$ Cen (Sollima et al. 2009) and $[\mathrm{Fe} / \mathrm{H}]=-2.0$ for NGC 6397 (Carretta et al. 2009), and we use color transformation tables provided by Lejeune et al. (1998). The parameters derived for our target stars from the observational data are given in Table 2.

The last column in Table 2 contains mass-center velocities with respect to the cluster obtained by a simple sinusoid fit, with heliocentric velocities of the clusters $\left(232.3 \mathrm{~km} \mathrm{~s}^{-1}\right.$ for $\omega$ Cen and $18.9 \mathrm{~km} \mathrm{~s}^{-1}$ for NGC 6397) taken from the catalog compiled by Harris (1996) and updated online ${ }^{2}$. The velocities of V214, V240, and V254 may seem a bit large for their distances from the cluster center $\left(17^{\prime} .0,14^{\prime} .9\right.$, and $14{ }^{\prime} .7$, respectively), even though the published cluster tidal radius is $r_{\mathrm{t}}=44.8$ (Trager et al. 1995), or $r_{\mathrm{t}}=57.03$ according to the Harris (1996) cata$\log$. That these stars are well within the limits for cluster membership is confirmed by Fig. 6 in Sollima et al. (2009), which shows the variation in radial velocity of $\omega$ Cen stars as a function of distance from the cluster center. Still, V240 turned out to be a doubtful case (see Sect. 3.3.1). As for V214 and V254, we checked that the best fits described in Sect. 3.3.1 were not affected when $v_{0}$ was lowered, respectively, to 10 and $6 \mathrm{~km} \mathrm{~s}^{-1}$. The velocity of NV360, although also rather large, is entirely consistent with the distance of this system from the center of $\omega$ Cen (3'.6), at which the relative velocities of cluster members reach $\pm 35 \mathrm{~km} \mathrm{~s}^{-1}$ (Sollima et al. 2009).

${ }^{2}$ http://www.physics.mcmaster.ca/Globular.html 
Table 2. Parameters of the target objects derived from the observations.

\begin{tabular}{lcclrrrr}
\hline \hline Cluster & Star & $\begin{array}{c}P \\
{[\mathrm{~d}]}\end{array}$ & $\begin{array}{l}M_{\mathrm{bol}}^{\mathrm{o}} \\
{[\mathrm{mag}]}\end{array}$ & $\begin{array}{c}T \\
{[\mathrm{~K}]}\end{array}$ & $\begin{array}{r}K_{\text {obs }} \\
{\left[\mathrm{km} \mathrm{s}^{-1}\right]}\end{array}$ & $\begin{array}{r}f_{m} \\
{\left[M_{\odot}\right]}\end{array}$ & $\begin{array}{r}v_{0} \\
{\left[\mathrm{~km} \mathrm{~s}^{-1}\right]}\end{array}$ \\
\hline$\omega$ Cen & V214 & 0.341806 & 2.44 & 8300 & 35 & $1.5 \times 10^{-3}$ & 21 \\
$\omega$ Cen & V240 & 0.331888 & 2.19 & 8000 & $<10$ & $<3.4 \times 10^{-5}$ & 21 \\
$\omega$ Cen & V251 & 0.922458 & 2.06 & 8300 & 35 & $4.1 \times 10^{-3}$ & 6 \\
$\omega$ Cen & V254 & 0.385071 & 2.30 & 8300 & 60 & $8.0 \times 10^{-3}$ & 18 \\
$\omega$ Cen & NV334 & 0.257881 & 4.52 & 7300 & 35 & $1.1 \times 10^{-3}$ & -9 \\
$\omega$ Cen & NV360 & 0.630821 & 2.98 & 8300 & 45 & $5.9 \times 10^{-3}$ & -17 \\
$\omega$ Cen & NV400 & 0.636874 & 2.40 & 8800 & 20 & $5.3 \times 10^{-4}$ & -7 \\
NGC 6397 & V17 & 1.061316 & 3.35 & 6200 & 50 & $1.4 \times 10^{-2}$ & -2 \\
NGC 6397 & V20 & 0.861177 & 2.94 & 7200 & $*$ & & 9 \\
NGC 6397 & V27 & 0.556134 & 5.38 & 7500 & 25 & $9.0 \times 10^{-4}$ & -6 \\
NGC 6397 & V36 & 1.098569 & 3.65 & 6200 & 95 & $9.7 \times 10^{-2}$ & -1 \\
\hline
\end{tabular}

Notes. ${ }^{(*)}$ Velocities of both components were measured, yielding $K_{1}=25 \mathrm{~km} \mathrm{~s}^{-1}$ and $K_{2}=130 \mathrm{~km} \mathrm{~s}^{-1}$. The corresponding mass functions are $f_{m 1}=1.4 \times 10^{-3} M_{\odot}$ and $f_{m 2}=1.9 \times 10^{-1} M_{\odot}$.

With $T$ fixed, the task is to find the orbital separation $a$, inclination of the orbit $i$, and masses of the components $m_{1}$ and $m_{2}$. Unless indicated otherwise, we assume that the system is semidetached, with the visible component filling its Roche lobe. It must be stressed here that this particular model of the binary is not related to the physical structure of our targets (in fact, in some cases it might cause the mass-transfer to be unstable on a dynamical scale). A semi-detached system is simply the most compact one among those with $M_{\mathrm{bol}}^{\mathrm{c}}=M_{\mathrm{bol}}^{\mathrm{o}}$. Focusing on it, we minimize the orbital separation, so that the corresponding "semidetached" masses $m_{1}^{\text {sd }}$ and $m_{2}^{\text {sd }}$ are the lowest allowable given $P$ and $q$. The lower limits of the actual masses of the components are obtained by finding "the best" mass ratio $q_{b}$, defined as the one for which $\Delta V_{\mathrm{c}} / \Delta V_{\mathrm{obs}}=1$ (see Sect. 3.2 for further discussion). Our analysis involves the following steps:

1. specify if the visible component is primary or secondary;

2. set the mass ratio $q \equiv m_{2} / m_{1}<1$ (we define the primary as the more massive component, not necessarily the more luminous one);

3. given the orbital period $P$, adjust the separation $a$ so that the calculated bolometric magnitude $M_{\mathrm{bol}}^{\mathrm{c}}$ of the visible component equal to $M_{\mathrm{bol}}^{\mathrm{o}}$;

4. adjust $i$ and $v_{0}$ so that the calculated velocity curve fits the observed one, and find the ratio of the computed amplitude of the light curve $\left(\Delta V_{\mathrm{c}}\right)$ to the observed amplitude $\left(\Delta V_{\text {obs }}\right)$;

5. repeat steps (2)-(4) for several $q$ values;

6. if in step (1) the visible component was specified as the primary, specify it as the secondary (and vice-versa). Repeat steps (2)-(4) for several values of $q$.

The calculations in steps (3) and (4) are performed using the PHOEBE interface (Prša \& Zwitter 2005) to the Wilson-Devinney code (Wilson \& Devinney 1971).

In principle, using a relation between $V, B-V$ and stellar angular diameter $\theta$ (Kervella et al. 2004) would be more straightforward and simpler than calculating $M_{\mathrm{bol}}^{\mathrm{o}}$ and $T$, and comparing $M_{\mathrm{bol}}^{\mathrm{o}}$ to $M_{\mathrm{bol}}^{\mathrm{c}}$. ( $T$ is needed for PHOEBE to derive $M_{\mathrm{bol}}^{\mathrm{c}}$ ) Based on such a relation, we could directly fit the "observed" radius of the visible component $R^{\circ} \equiv \theta D$ (where $D$ is the distance to the cluster) to the calculated average radius of the Roche lobe. Unfortunately, fits of Kervella et al. (2004) proved to be unreliable when extrapolated to low metallicity and small $\theta$. This is not surprising, as these authors explicitly warn about the nonlinearity of the relation involving $V$ and $B-V$.

\subsection{Discussion of errors}

The values of our key input parameters, i.e. temperatures and bolometric luminosities, are not known accurately, and their errors are difficult to estimate without engaging in a lengthly (and likely ambiguous) discussion of involved factors. In order to verify how these uncertainties influence the output we varied $T$ and $M_{\text {bol }}^{\mathrm{o}}$ by small amounts and observed the corresponding variations in $m_{1}$ and $m_{2}$. We found that decreasing $T$ by $100 \mathrm{~K}$ causes the masses to grow by $7-10 \%$, while an increase in $M_{\mathrm{bol}}^{\mathrm{o}}$ by -0.1 mag makes them larger by $10-15 \%$.

At a first glance, focusing on semi-detached systems seems too restrictive. To see it clearly, suppose that the visible component is entirely contained within its Roche lobe. Then, to recover $M_{\mathrm{bol}}^{\mathrm{o}}$, we would have to increase the orbital separation $a$. Since the period $P$ is fixed, the masses would have to increase, too. To keep the observed velocity amplitude constant, the inclination $i$ would have to decrease. For that reason, and also because the visible component would now be less deformed, $\Delta V_{\mathrm{c}}$ would also decrease. This way the range of $q$ for which the ratio $\Delta V_{\mathrm{c}} / \Delta V_{\mathrm{obs}} \approx 1$ could be extended onto fits that for semidetached systems yield $\Delta V_{\mathrm{c}} / \Delta V_{\mathrm{obs}}>1$. In principle, it is even possible that $m_{1}$ and $m_{2}$ derived for some mass ratio $q \neq q_{b}$ from detached fits could be smaller than $m_{1}^{\text {sd }}\left(q_{b}\right)$ and $m_{2}^{\text {sd }}\left(q_{b}\right)$. However, upon verifying this possibility we found that in most cases the detached fit results in either too low an amplitude for the light-curve or masses higher than those obtained for $q_{b}$, so that the extension of the $q$-range is marginal (if any). We conclude that the errors in the mass limits caused by neglecting detached configurations are smaller than those related to bolometric corrections or the color-temperature scale.

Another possible source of errors is the assumption that the whole light output of the system originates in the component responsible for the observed spectrum. The components of the only system in our sample in which two sets of velocities could be measured differ in brightness by $\Delta M \approx 1 \mathrm{mag}$. Assuming that in the remaining systems $\Delta M=2$ mag causes their brighter components to be dimmer by $0.16 \mathrm{mag}$. The corresponding decrease in masses, estimated from the general relation between mass and $M_{\mathrm{bol}}^{\mathrm{o}}$ mentioned earlier, amounts to $15-20 \%$.

\subsection{Results}

Quantitative results of the analysis are shown in Tables 3-13 and discussed in Sect. 3.3. The best fits are indicated with an asterisk 
Table 3. Derived parameters for V214.

\begin{tabular}{cccccc}
\hline \hline$q$ & $a$ & $i$ & $m_{1}$ & $m_{2}$ & $\Delta V_{\mathrm{c}} / \Delta V_{\mathrm{obs}}$ \\
& {$\left[R_{\odot}\right]$} & {$\left[{ }^{\circ}\right]$} & {$\left[M_{\odot}\right]$} & {$\left[M_{\odot}\right]$} & \\
\hline 0.10 & 2.45 & 62 & 1.54 & 0.15 & 1.1 \\
0.13 & 2.55 & 58 & 1.69 & 0.22 & $1.0^{*}$ \\
0.15 & 2.60 & 47 & 1.76 & 0.26 & 0.6 \\
\hline
\end{tabular}

Table 4. Derived parameters for V251.

\begin{tabular}{cccccc}
\hline \hline$q$ & $a$ & $i$ & $m_{1}$ & $m_{2}$ & $\Delta V_{\mathrm{c}} / \Delta V_{\mathrm{obs}}$ \\
& {$\left[R_{\odot}\right]$} & {$\left[{ }^{\circ}\right]$} & {$\left[M_{\odot}\right]$} & {$\left[M_{\odot}\right]$} & \\
\hline 0.20 & 3.10 & 33 & 0.39 & 0.08 & 3.2 \\
0.35 & 3.44 & 32 & 0.48 & 0.17 & $1.0^{*}$ \\
0.50 & 3.67 & 22 & 0.52 & 0.26 & 0.65 \\
\hline
\end{tabular}

in the last column of each table, and the corresponding computed light and velocity curves are displayed in Figs. 3-6, along with the observational data.

\subsection{1. $\omega$ Centauri}

V214. Assuming that the visible component is the secondary results in computed light-curve amplitudes that are consistently much too low. Assuming that it is the primary makes the difference between $\Delta V_{\text {obs }}$ and $\Delta V_{\mathrm{c}}$ smaller, but still unacceptable.

To make $\Delta V_{\mathrm{c}}$ larger, we are forced to look for solutions in which both components contribute to the observed ellipticity effect. Based on the simplest possible assumption that the system is in contact, we apply the following procedure: i) set $q$; ii) adjust $a$ so that the combined $M_{\mathrm{bol}}$ of the system is $2.44 \mathrm{mag}$; iii) adjust $i$ so that the computed velocity curve agrees with the observed one; iv) check if the computed light curve agrees with the observed one.

The fits are shown in Table 3, and we conclude that the available data favor a rather high mass ratio, indicating that the system has undergone significant mass transfer (and may still be transferring mass at a low rate). Obviously, the sum $m=m_{1}+m_{2}$ should not exceed two turnoff masses of the youngest population of $\omega$ Cen, i.e. $1.84 M_{\odot}$ (Norris 2004). Given the rather large errors in the mass estimates due to uncertainties in $T$ and $M_{\mathrm{bol}}^{\mathrm{o}}$, one may accept that this requirement is fulfilled by at least the first two fits in Table 3.

V251. As in the previous cases, by assuming that the spectrum originates in the secondary we find that for all $q$ values $\Delta V_{\mathrm{c}}$ is much smaller than $\Delta V_{\text {obs }}$. Assigning the spectrum to the primary leads to the results displayed in Table 4. As the total mass is markedly lower than $1.84 M_{\odot}$, we seem to have a system that not only underwent mass transfer, but also lost a significant amount of mass.

V254. Assuming that the spectrum originates in the secondary leads once more to amplitude ratios $\Delta V_{\mathrm{c}} / \Delta V_{\text {obs }}$, which for all mass ratios are much less than unity. Upon adopting that the source of the spectrum is the primary, we get the results displayed in Table 5, which suggests that V254 is similar to V214. NV334. Because of the low brightness of the system $(V=$ $18.83 \mathrm{mag}$ ) we were only able to obtain a very rough estimate of the velocity amplitude. We therefore modifed our analysis by estimating the inclination based on the light curve and comparing the calculated velocity amplitude $K_{\mathrm{c}}$ to the observed one. Assuming that the spectrum originates in the secondary leads to $K_{\mathrm{c}}$ being much larger than $K_{\mathrm{obs}}$, regardless of the value of $q$.
Table 5. Derived parameters for V254.

\begin{tabular}{cccccc}
\hline \hline$q$ & $a$ & $i$ & $m_{1}$ & $m_{2}$ & $\Delta V_{\mathrm{c}} / \Delta V_{\text {obs }}$ \\
& {$\left[R_{\odot}\right]$} & {$\left[{ }^{\circ}\right]$} & {$\left[M_{\odot}\right]$} & {$\left[M_{\odot}\right]$} & \\
\hline 0.2 & 2.77 & 72 & 1.61 & 0.32 & 1.7 \\
0.3 & 2.98 & 43 & 1.84 & 0.55 & $1.0^{*}$ \\
0.5 & 3.29 & 24 & 2.15 & 1.07 & 0.6 \\
\hline
\end{tabular}

Table 6. Derived parameters for NV334.

\begin{tabular}{cccccc}
\hline \hline$q$ & $a$ & $i$ & $m_{1}$ & $m_{2}$ & $K_{\mathrm{c}} / K_{\mathrm{obs}}$ \\
& {$\left[R_{\odot}\right]$} & {$\left[{ }^{\circ}\right]$} & {$\left[M_{\odot}\right]$} & {$\left[M_{\odot}\right]$} & \\
\hline 0.1 & 1.22 & 76 & 0.33 & 0.03 & 0.4 \\
0.3 & 1.45 & 65 & 0.47 & 0.14 & $1.2^{*}$ \\
0.5 & 1.60 & 63 & 0.55 & 0.27 & 1.8 \\
\hline
\end{tabular}

Table 7. Derived parameters for NV360.

\begin{tabular}{cccccc}
\hline \hline$q$ & $\begin{array}{c}a \\
{\left[R_{\odot}\right]}\end{array}$ & $\begin{array}{c}i \\
{\left[{ }^{\circ}\right]}\end{array}$ & $\begin{array}{c}m_{1} \\
{\left[M_{\odot}\right]}\end{array}$ & $\begin{array}{c}m_{2} \\
{\left[M_{\odot}\right]}\end{array}$ & $K_{\mathrm{c}} / K_{\mathrm{obs}}$ \\
\hline 0.5 & 2.52 & 26 & 0.36 & 0.18 & 0.7 \\
0.8 & 2.79 & 25 & 0.41 & 0.32 & $1.0^{*}$ \\
0.9 & 2.86 & 25 & 0.42 & 0.37 & 1.1 \\
\hline
\end{tabular}

Table 8. Derived parameters for NV400.

\begin{tabular}{cccccc}
\hline \hline$q$ & $\begin{array}{c}a \\
{\left[R_{\odot}\right]}\end{array}$ & $\begin{array}{c}i \\
{\left[{ }^{\circ}\right]}\end{array}$ & $\begin{array}{c}m_{1} \\
{\left[M_{\odot}\right]}\end{array}$ & $\begin{array}{c}m_{2} \\
{\left[M_{\odot}\right]}\end{array}$ & $\Delta V_{\mathrm{c}} / \Delta V_{\mathrm{obs}}$ \\
\hline 0.3 & 2.65 & 28 & 0.48 & 0.14 & 1.3 \\
0.5 & 2.93 & 13 & 0.56 & 0.28 & $0.8^{*}$ \\
0.8 & 3.24 & 9 & 0.63 & 0.50 & 0.2 \\
\hline
\end{tabular}

The same analysis applied to the configuration with the primary generating the spectrum produces the results shown in Table 6, which seem to indicate that NV334 is similar to V251. However, the similarity is superficial: the effective temperatures of the two systems differ by $\sim 4000 \mathrm{~K}$, causing the cooler and dimmer NV334 to occupy an entirely different position in the colormagnitude diagram of $\omega$ Cen (see Fig. 1).

NV360. Our standard analysis fails for this system (iterations of the inclination $i$ based on the velocity curve are either converging extremely slowly or entirely diverging), so that we again have to apply a modified version based on the light curve. As for NV334, assuming that the spectrum originates in the secondary leads to $K_{\mathrm{c}}$ being much larger than $K_{\mathrm{obs}}$, regardless of the value of $q$. Systems with the primary responsible for the spectrum fare much better (see Table 7), and we conclude that NV360, unlike NV334, is truly similar to NV251.

NV400. When the secondary is assumed to generate the spectrum, the calculated light-curve amplitude is for all values of $q$ much higher than the observed one. Assuming that the spectrum originates in the primary, we get the results displayed in Table 8. Apparently, NV400 is yet another system similar to V251.

V240. Assuming that the spectrum originates in the primary, we obtain the results shown in Table 9: computed light-curve amplitudes are far too low. If the secondary were the source of the spectrum they would be even lower. Moreover, the total mass of the system is unacceptably high. (As we argued in the case of V214, it should not exceed $1.84 M_{\odot}$.) In other words, the color and apparent magnitude of V240 are incompatible with its observed velocity amplitude, suggesting that this system does not belong to $\omega$ Cen. Another possibility is that the light of V240 is 
Table 9. Derived parameters for V240.

\begin{tabular}{cccccc}
\hline \hline$q$ & $a$ & $i$ & $m_{1}$ & $m_{2}$ & $\Delta V_{\mathrm{c}} / \Delta V_{\mathrm{obs}}$ \\
& {$\left[R_{\odot}\right]$} & {$\left[{ }^{\circ}\right]$} & {$\left[M_{\odot}\right]$} & {$\left[M_{\odot}\right]$} & \\
\hline 0.07 & 2.83 & 16 & 2.59 & 0.18 & 0.1 \\
0.1 & 2.96 & 11 & 2.88 & 0.29 & 0.05 \\
\hline
\end{tabular}
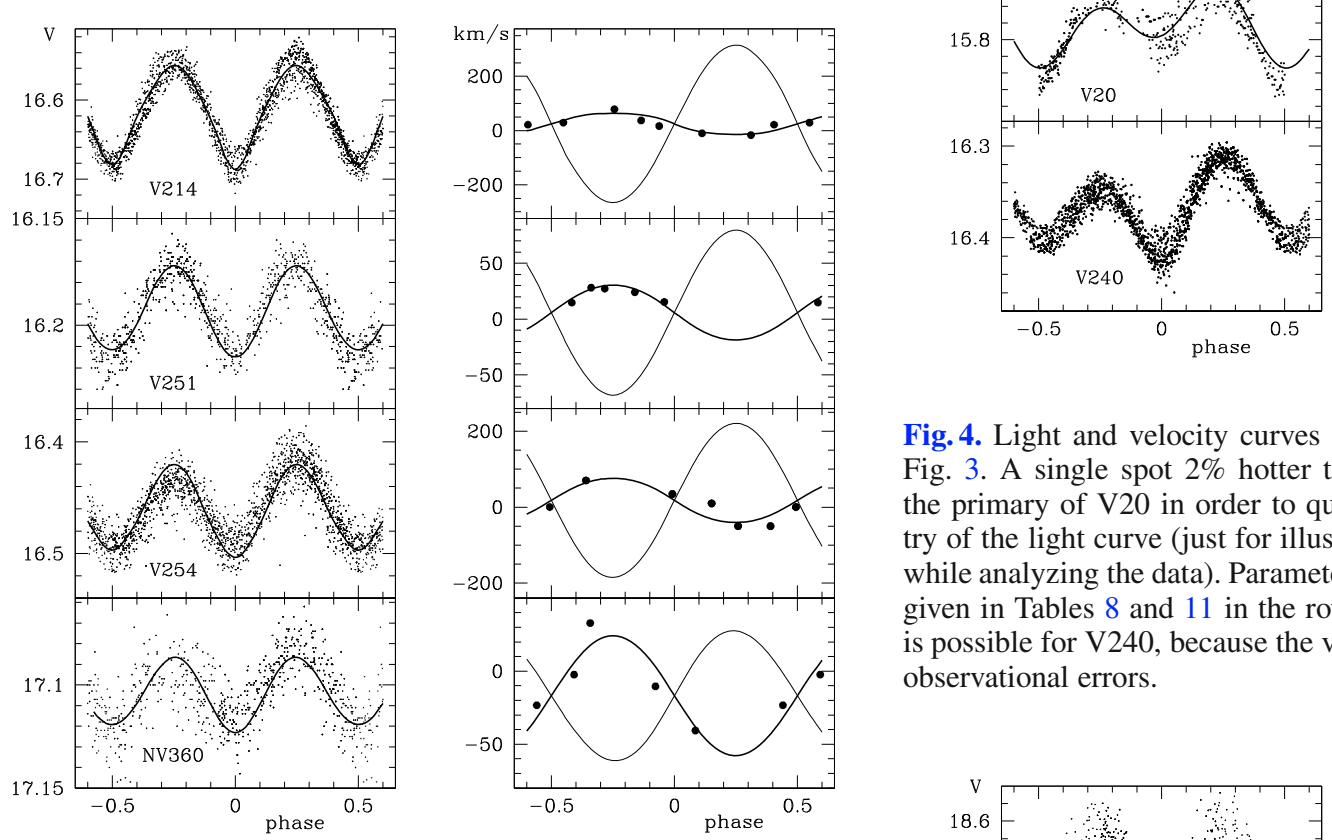
observational errors.

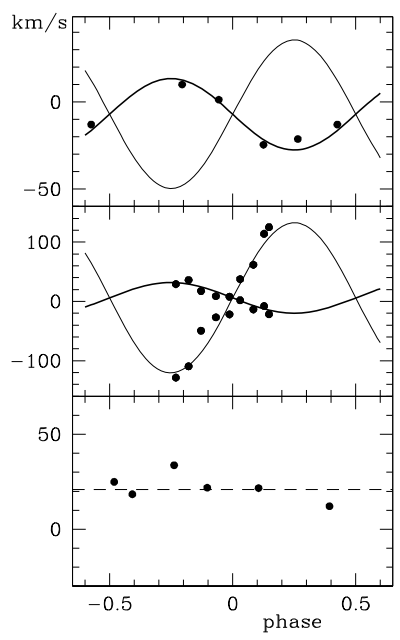

Fig. 4. Light and velocity curves of blue stragglers - continuation of Fig. 3. A single spot $2 \%$ hotter than the photosphere was placed on the primary of V20 in order to qualitatively account for the asymmetry of the light curve (just for illustrative purposes; it was not included while analyzing the data). Parameters of the fits for NV400 and V20 are given in Tables 8 and 11 in the rows indicated with an asterisk. No fit is possible for V240, because the velocity amplitude is smaller than the
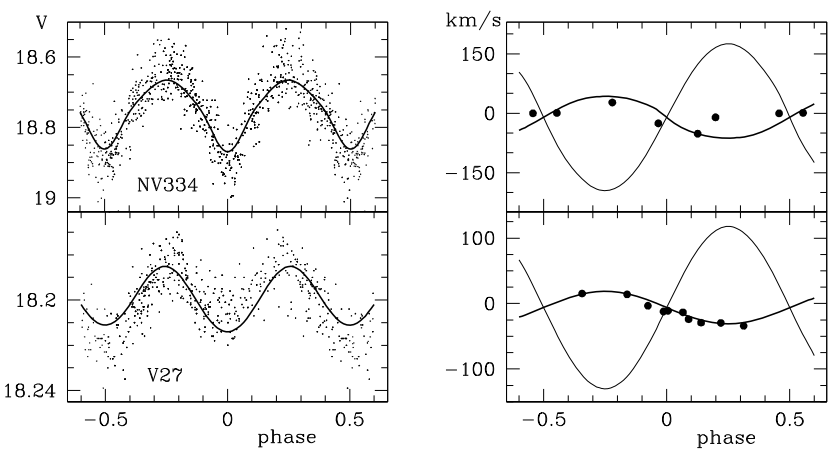

Fig. 5. Light and velocity curves of subdwarfs. Parameters of the plotted fits are given in Tables 6 and 12 in the rows indicated with an asterisk.

Table 10. Derived parameters for V17.

\begin{tabular}{cccccc}
\hline \hline$q$ & $a$ & $i$ & $m_{1}$ & $m_{2}$ & $\Delta V_{\mathrm{c}} / \Delta V_{\mathrm{obs}}$ \\
& {$\left[R_{\odot}\right]$} & {$\left[{ }^{\circ}\right]$} & {$\left[M_{\odot}\right]$} & {$\left[M_{\odot}\right]$} & \\
\hline 0.3 & 6.00 & 14 & 1.99 & 0.60 & 0.7 \\
0.4 & 5.56 & 16 & 1.47 & 0.59 & 0.9 \\
0.5 & 5.25 & 19 & 1.15 & 0.58 & $1.1 *$ \\
0.6 & 5.02 & 21 & 0.95 & 0.57 & 1.5 \\
\hline
\end{tabular}

Since the mass ratio is low $(q \approx 0.2)$, the secondary must be oversized for its mass. We assume that it fills its Roche lobe, and we apply the following nonstandard procedure: i) set $i$; ii) iterate $a$ and $q$ to fit the velocity curves of both components; iii) adjust the size of the primary to get $M_{\text {bol1 }}^{\mathrm{c}}=3.31 \mathrm{mag}$; iv) adjust the temperature of the secondary $T_{2}$ to get $M_{\mathrm{bol} 2}^{\mathrm{c}}=4.29 \mathrm{mag}$ (this is the minimum possible temperature). The best fit among those displayed in Table 11 indicates that V20 is another system similar to V251.
0.02 which, combined with $M_{\mathrm{o}}^{\mathrm{o}}=2.94 \mathrm{mag}$, yields $M_{\mathrm{bol}}^{\mathrm{o}}=$ $3.31 \mathrm{mag}$ and $M_{\mathrm{bol} 2}^{\mathrm{o}}=4.29 \mathrm{mag}$. 

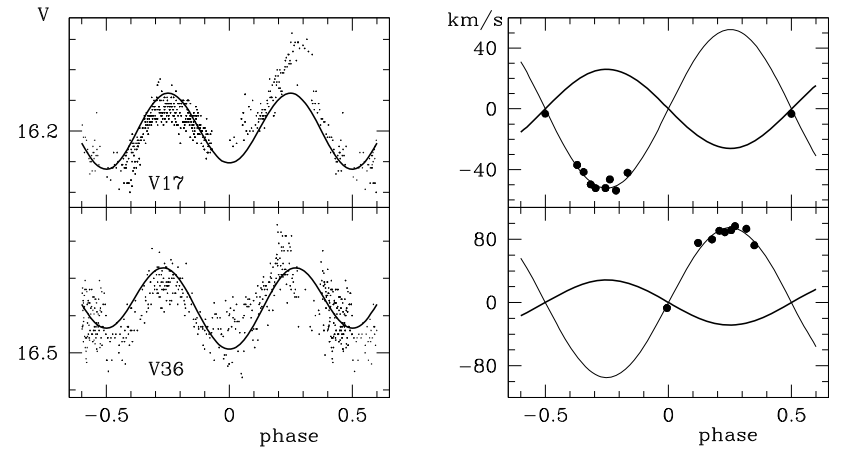

Fig. 6. Light and velocity curves of systems at the main sequence turnoff. Small ticks in light-curve plots are separated by 0.01 mag. Parameters of the plotted fits are given in Tables 10 and 13 in the rows indicated with an asterisk.

Table 11. Derived parameters for V20.

\begin{tabular}{cccccc}
\hline \hline$i$ & $\begin{array}{c}a \\
{\left[R_{\odot}\right]}\end{array}$ & $\begin{array}{c}q \\
{\left[{ }^{\circ}\right]}\end{array}$ & $\begin{array}{c}m_{1} \\
{\left[M_{\odot}\right]}\end{array}$ & $\begin{array}{c}m_{2} \\
{\left[M_{\odot}\right]}\end{array}$ & $\Delta V_{\mathrm{c}} / \Delta V_{\text {obs }}$ \\
\hline 30 & 5.28 & 0.20 & 2.23 & 0.45 & 0.4 \\
40 & 4.09 & 0.20 & 1.04 & 0.20 & 0.8 \\
45 & 3.72 & 0.20 & 0.78 & 0.15 & $1.0^{*}$ \\
50 & 3.43 & 0.20 & 0.61 & 0.12 & 1.2 \\
\hline
\end{tabular}

Table 12. Derived parameters for V27.

\begin{tabular}{ccccccc}
\hline \hline$q$ & $a$ & $i$ & $m_{1}$ & $m_{2}$ & $T_{2}$ & $\Delta V_{\mathrm{c}} / \Delta V_{\mathrm{obs}}$ \\
& {$\left[R_{\odot}\right]$} & {$\left[{ }^{\circ}\right]$} & {$\left[M_{\odot}\right]$} & {$\left[M_{\odot}\right]$} & & \\
\hline 0.2 & 2.00 & 55 & 0.29 & 0.06 & 6500 & $1.0^{*}$ \\
0.2 & 2.50 & 40 & 0.57 & 0.11 & 6000 & 0.9 \\
0.2 & 3.00 & 33 & 0.98 & 0.20 & 6000 & 0.3 \\
0.3 & 1.80 & 43 & 0.20 & 0.06 & 6000 & 0.9 \\
0.3 & 2.00 & 36 & 0.27 & 0.08 & 6000 & 0.7 \\
0.3 & 2.50 & 28 & 0.52 & 0.17 & 6000 & 0.2 \\
\hline
\end{tabular}

V27. Regardless of whether the primary or the secondary produces the spectrum the standard analysis indicates that for a given value of $q$ either there is no inclination for which the calculated amplitude matches the observed one or the masses of both stars are unacceptably low $\left(m_{1} \leq 0.05 M_{\odot} ; m_{2} \leq 0.04 M_{\odot}\right)$. One can make them higher by assuming that the system is detached instead of semi-detached; however, in such a case it is impossible to find firm lower mass limits.

To obtain a sample of detached solutions we modified our standard procedure in the following way: i) set $q$ and $a$; ii) adjusted $i$ so that the calculated velocity curve agrees with the observed one; iii) adjusted the size of the primary to get $M_{\text {boll }}=$ $6.0 \mathrm{mag}$; iv) adjusted the size (and, if necessary, the temperature) of the secondary to get $M_{\mathrm{bol} 2}=6.3 \mathrm{mag}^{3}$; v) check that the calculated light curve agrees with the observed one.

If one assumes that the spectrum is generated by the secondary, then the inclination consistent with the velocity curve is much too low to reproduce the light curve (except for systems with $q \gtrsim 0.75$, but in those cases the masses of the components are lower than $0.05 M_{\odot}$ ). The only reasonable solutions we were able to find for the detached configuration are based

\footnotetext{
3 These values are of course arbitrary, the only constraints being that the secondary is not much dimmer than the primary, and the combined bolometric magnitude of the system is $5.38 \mathrm{mag}$.
}

Table 13. Derived parameters for V36.

\begin{tabular}{cccccc}
\hline \hline$q$ & $a$ & $i$ & $m_{2}$ & $m_{1}$ & $\Delta V_{\mathrm{c}} / \Delta V_{\text {obs }}$ \\
& {$\left[R_{\odot}\right]$} & {$\left[{ }^{\circ}\right]$} & {$\left[M_{\odot}\right]$} & {$\left[M_{\odot}\right]$} & \\
\hline 0.2 & 7.40 & 20 & 0.75 & 3.77 & 0.9 \\
0.3 & 6.62 & 24 & 0.75 & 2.49 & $1.0^{*}$ \\
0.4 & 6.17 & 28 & 0.75 & 1.87 & 1.3 \\
0.5 & 5.86 & 32 & 0.75 & 1.50 & 2.1 \\
\hline
\end{tabular}

on the assumption that the primary generates the observed spectrum, while the secondary is mainly responsible for the observed ellipticity effect. The results shown in Table 12 seem to favor a configuration with low and rather discrepant masses.

V36. The visible component must be the secondary. The alternative assumption enforces inclinations such that the calculated amplitude of the light curve is much lower than the observed one for all values of $q$. However, assuming that the secondary fills its Roche lobe is untenable, as our standard analysis indicates that in such a case the calculated amplitudes of the light curve are too high. Thus, we are forced to adopt a detached configuration. Since V36 is located at the turnoff (only 0.3 mag below V17; see Fig. 2), we assume that $m_{2}=0.75 M_{\odot}$ and apply the following four-step procedure: i) set $q$; ii) adjust $a$ to get $m_{2}=0.75 M_{\odot}$; iii) adjust $i$ so that the calculated velocity curve agrees with the observed one; iv) adjust the size of the secondary to get $M_{\text {bol2 }}=3.65 \mathrm{mag}$. The results shown in Table 13 indicate that V36 is similar to V17, with the primary's mass possibly even higher than $1.5 M_{\odot}$.

\section{Discussion and conclusions}

Ten out of 11 objects in our sample exhibit radial-velocity variations, indicating their binary nature (the only exception is V240 in $\omega$ Cen). In that sense, the sample was well chosen. Regarding the principal aim of the present survey we have been less successful, because no clear-cut evidence of high-mass degenerate components was found. However, while 8 systems proved to be more or less ordinary binaries, the remaining two (V17 and V36 at the turnoff of NGC 6397) cearly deserve further scrutiny. First, they are the only systems in which the brighter component is the less massive secondary. Second, the masses of their dim primaries may be significantly higher than $1 M_{\odot}$ (in V36, even higher than $2 M_{\odot}$ if the secondary is indeed a turnoff-mass star). Third, they are weak X-ray sources. Based on Chandra observations, Bogdanov et al. (2010) classify them as active binaries $(\mathrm{AB})$, i.e. systems composed of main-sequence or subgiant stars whose weak X-ray emission originates from magnetic activity. Our results rule out the possibility that they are composed of pristine stars which have not undergone any mass transfer episode, as in such a case the more massive component would also have to be the brighter one. This is not to say Bogdanov et al. (2010) are wrong: short-period degenerate binaries in quiescence may easily "masquerade" as AB systems, since they are most likely synchronized and their visible components spin fast enough for the stellar dynamo to be highly efficient.

According to the most optimistic interpretation of our results, V17 and V36 may contain a neutron star (the dim primary of V36 may even be a black hole). Such systems can indeed be expected, as population synthesis calculations indicate that the formation of a degenerate binary in which a neutron star or a black hole is accompanied by a main-sequence star or a subgiant is nothing unusual in globular clusters. Although the rate at which they form is not high $(<2.5$ systems/Gyr), 
they should be transient all the time, and therefore more likely seen as a qLMXBs rather than as bright LMXBs (Ivanova et al. 2008). Moreover, NGC 6397 belongs to core-collapse clusters for which the formation rates of Ivanova et al. (2008) are just lower limits. Another (admittedly, not too strong) support for the optimism comes from the detection of a possible qLMXB near the turnoff of M30 (Lugger et al. 2006). Main-sequence secondaries similar to those in V17 and V36 are also found in galactic $\mathrm{X}$-ray binaries whose primary components are black hole candidates; e.g. V1033 Sco, GRS 1739-278 or V821 Ara (Ziolkowski, priv. comm).

That all our blue stragglers except V240 are binary supports the currently leading hypothesis concerning the nature of these objects, namely that they result rather from an extensive mass exchange between the component stars than from stellar collisions (Knigge et al. 2009). In all cases, the brighter component is the primary that, according to the mass-transfer scenario, must have acquired significant amounts of hydrogen-rich material from the envelope of the originally more massive secondary. In fact, our results seem to indicate that blue stragglers are Algol-like systems in which the original mass ratio has been reversed, causing the mass transfer to effectively stop. Our stragglers may be similar to the well-studied star V228 in 47 Tuc (Kaluzny et al. 2007); however, we cannot exclude the possibility that in some of them the primary is already at the beginning of the subgiant branch and has become large enough to approach its Roche lobe.

V214 and V254 in $\omega$ Cen have primaries with masses likely exceeding $1 M_{\odot}$ (maybe even $1.5 M_{\odot}$ ), and their total masses approach $2 M_{\odot}$. Systems that massive must have evolved from binaries subject to conservative or nearly conservative mass transfer. They also have large amplitudes of light variations (0.12, mag and $0.10 \mathrm{mag}$, respectively), in our sample rivaled only by that of NV334 ( 0.3 mag); however, a significant contribution to this effect must originate from relatively large inclinations found for all three systems. The remaining blue stragglers are significantly less massive - their total masses are lower than $1 M_{\odot}$. As far as we know, our results are the first to indicate such a broad (and possibly bimodal) mass distribution. If this effect is real, it probably reflects differences in the efficiency of mass transfer and/or mass loss. We note in passing that some mass may still be flowing between the components of V20, as this system exhibits an asymmetric and variable light curve, and is a weak X-ray source. Another possibility to account for the apparent large width of the mass distribution is related to the peculiarities of the chemical composition of $\omega$ Cen, in which strongly He-enriched subpopulations are suggested to exist, with $Y$ reaching up to $\sim 0.4$ (e.g., Norris 2004; Piotto et al. 2005; but see also Catelan et al. 2010, for a recent review and some caveats). Our fits are based on the standard value $Y=0.24$, and for a given $B-V$ color they can overestimate the temperature of enriched stars by up to a few hundred Kelvin (Dotter et al. 2008). If this is what happened, then our standard analysis caused the He-rich systems to artificially shrink both in size and mass. Such hypothesis would also explain why stragglers with signficantly different "apparent" masses are able to form a relatively tight group on the H-R diagram.

The subdwarfs NV334 and V27 are very similar to each other in that they have high mass ratios and low-mass primaries with practically the same effective temperatures. Our estimate of the primary's mass in NV334 is consistent with the canonical hot subdwarf mass of $0.47 M_{\odot}$, while the estimated mass of the secondary falls near the peak of the mass distribution of the unseen companions to field hot subdwarf stars (Geier et al. 2009). On the other hand, and as pointed out by
Moni Bidin et al. (2008), the close binary fraction among subdwarf stars appears to be much lower in GCs than in the field, which may point to different formation mechanisms for many of the GC subdwarfs. In V27 the estimated masses are significantly lower (see Table 12); however, they are also less reliable due to problems discussed in Sect. 3.3.2, and the solution with $m_{1}=$ $0.57 M_{\odot}$ and $m_{2}=0.11 M_{\odot}$ is also acceptable. Taken at face value, the starred parameters in Table 12 would indicate that V27 is very similar to HS $2231+2241$, an HW Vir-type system with a companion at or below the lower mass-limit for M-dwarfs. The $\sim 0.26 M_{\odot}$ primary of HS $2231+2241$ is a non-helium burning, post-RGB star (Østensen et al. 2008). Another example of such a star is the $0.24 M_{\odot}$ component of HD 188112 (Heber et al. 2003). The primaries of NV334 and V27 are much cooler $(T \sim 7500 \mathrm{~K}$ compared to $T=28400 \mathrm{~K}$ and $T=20500 \mathrm{~K}$, respectively, for HS 2231+2241 and HD 188112), but this difference may result from their more advanced evolutionary stage.

Our sample is too small for a meaningful statistical analysis; nevertheless, the results discussed above indicate that further research on ellipsoidal binaries in globular clusters is a wortwhile task, and suggest its most promising direction. It seems that future sampling of the photometric variables should be biased towards objects located either on or to the right of the main sequence, including those identified as weak X-ray sources. Obviously, for purely economical reasons (exposure times!) the targets sould not fall much below the turnoff point. Such an approach is tedious, but at the same time, it is the only one on which a fair census of degenerate binaries in globular clusters can be based.

Acknowledgements. We are very grateful to Jay Anderson for providing HST proper-motion data for NGC 6397 and to Janusz Ziolkowski for a primer on black hole candidates in galactic X-ray binaries. The research of J.K., P.P., and W.P. is supported by the grant MISTRZ from the Foundation for the Polish Science and by the grants N N203 379936 and N N203 301335 from the Polish Ministry of Science and Higher Education. Support for M.C. and C.C. is provided by MIDEPLAN's Programa Iniciativa Científica Milenio through grant P07-021-F, awarded to The Milky Way Millennium Nucleus; by Proyecto Basal PFB-06/2007; by FONDAP Centro de Astrofísica 15010003; and by Proyecto FONDECYT Regular \#1071002. IBT acknowledges the support of NSF grant AST-0507325. We sincerely thank the anonymous referee whose remarks significantly improved the presentation.

\section{References}

Bellini, A., Piotto, G., Bedin, L. R., et al. 2009, A\&A, 493, 959

Bogdanov, S., van den Berg, M., Heinke, C. O., et al. 2010, ApJ, 709, 241

Carretta, E., Bragaglia, A., Gratton, R., et al. 2009, A\&A, 508, 695

Catelan, M., Valcarce, A. A. R., \& Sweigart, A. V. 2010, in Star clusters: basic galactic building blocks, ed. R. de Grijs, \& J. R. D. Lépine, IAU Symp., 266, 281

Clark, G. W. 1975, ApJ, 199, L143

Devecchi, B., Colpi, M., Mapelli, M., et al. 2007, MNRAS, 380, 691 Dotter, A., Chaboyer, B., Jevremović, D., et al. 2008, ApJS, 178, 89

Downing, J. M. B., Benacquista, M. J., Giersz, M., \& Spurzem, R. 2010, MNRAS, in press [arXiv: 1008.5060 ]

Geier, S., Heber, U., Edelmann, H., et al. 2009, J. Phys. Conf. Ser., 172, 012008 Gendre, B., Barret, D., \& Webb, N. A. 2003, A\&A, 400, 521

Guillot, S., Rutledge, R. E., Bildsten, L., et al. 2009, MNRAS, 392, 665 Haggard, D., Cool, A. M., Anderson, J., et al. 2004, ApJ, 613, 512

Harris, W. E. 1996, AJ, 112, 1487

Heber, U., Edelmann, H., Lisker, T., \& Napiwotzki, R. 2003, A\&A, 411, L477 Ivanova, N., Heinke, C. O., Rasio, F. A., et al. 2008, MNRAS, 386, 553

Kaluzny, J., Olech, A., Thompson, I. B., et al. 2004, A\&A, 424, 1101

Kaluzny, J., Thompson, I. B., Krzeminski, W., et al. 2006, MNRAS, 365, 548

Kaluzny, J., Thompson, I. B., Rucinski, S. M., et al. 2007, AJ, 134, 541

Kaluzny, J., Thompson, I. B., Rucinski, S. M., \& Krzeminski, W. 2008, AJ, 136, 400

Katz, J. I. 1975, Nature, 253, 698

Kervella, P., Thévenin, F., Di Folco, E., \& Ségransan, D. 2004, A\&A, 426, 297 
M. Rozyczka et al.: A search for degenerate binaries in $\omega$ Centauri and NGC 6397

Knigge, C., Leigh, N., \& Sills, A. 2009, Nature, 457, 288

Lejeune, T., Cuisinier, F., \& Buser, R. 1998, A\&AS, 130, 65

Lugger, P. M., Cohn, H. N. Heinke, C. O., et al. 2006, ApJ, 657, 286

Lynch, R., Ransom, S. M., Lorimer, D., \& Boyles, J. 2010, BAAS, 42, 463

Maccarone, T. J., \& Servillat, M. 2008, MNRAS, 389, 379

Moni Bidin, C., Catelan, M., \& Altmann, M. 2008, A\&A, 480, L1

Munari, U., Sordo, R. M., Castelli, F., \& Zwitter, T. 2005, A\&A, 442, 1127

Norris, J. E. 2004, ApJ, 12, L25

Østensen, R. H., Oreiro, R., Hu, H., et al. 2008, ASPC, 392, 221

Piotto, G., Villanova, S., Bedin, L. R., et al. 2005, ApJ, 621, 777

Pooley, D. 2010, PNAS, 107, 7164

Prša, A., \& Zwitter, T. 2005, AJ, 628, 426

Remillard, R. A., \& McClintock, J. E. 2006, ARA\&A, 44, 49
Richer, H. B., Dotter, A., Hurley, J., et al. 2008, AJ, 135, 2141

Rucinski, S. M. 2002, AJ, 124, 1746

Sollima, A., Bellazzini, M., Smart, R. L., et al. 2009, MNRAS, 396, 2183

Stetson, P. B. 2000, PASP, 112, 925

Strickler, R. R., Cool, A. M., Anderson, J., et al. 2009, ApJ, 699, 40

Trager, S. C., King, I. R., \& Djorgovski, S. 1995, AJ, 109, 218; erratum: 1995, AJ, 109, 1912

van der Marel, R. P., \& Anderson, J. 2010, ApJ, 710, 1063

Verbunt, F., Pooley, D., \& Bassa, C. 2008, IAU Symp., 246, 301

Villanova, S., Piotto, G., King, I. R., et al. 2007, ApJ, 663, 296

Weldrake, D. T. F., Sackett, P. D., \& Bridges, T. J. 2007, AJ, 133, 1447

Wilson, R. E., \& Devinney, E. J. 1971, AJ, 166, 605

Zucker, S., \& Mazeh, T. 1994, ApJ, 420, 806 